\title{
Studies on Water Absorbency of Polyacrylamide
}

\section{Hydrogels}

\author{
Bhadani Reena $^{{ }^{*}}$ and Mitra Uttam Kumar ${ }^{2}$ \\ 1. Department of Chemistry, Ranchi Women's College, Ranchi University, Ranchi-834001, India \\ 2. Department of Chemistry, Gossner College, Ranchi University, Ranchi- 834001, India
}

\begin{abstract}
The polymerization was carried out under the different concentrations of acrylamide and crosslinker in an aqueous solution of $\mathrm{NO}_{2}$ gas as initiator at $50{ }^{\circ} \mathrm{C}$. The resulting polyacrylamide was saponified with $\mathrm{NaOH}$ solution. The hydrolysed polymers show high degree of water swelling $(>500)$ than that of unhydrolysed polymers $(<100)$. Swell ability of the gel decreases with the increasing concentration of N, N'- methylene bisacrylamide - a crosslinker which enhances the degree of crosslinking in the polymer chains. The increased crosslinking restricts the expansion of polymer network which causes the lowering of the water absorbency. It was observed that polymer hydration increased with the molar mass of polyacrylamide. The swell ability of polyacrylamide hydrogels is greatly lowered by the addition of ionic salts in the swelling medium. Both ionic strength and valency of the salts are responsible to decrease the water uptaking capacity of the gels which remain unaffected by nondessociating nature of urea. The hydrogels, so prepared, are stable and quite able to undergo swelling - deswelling cycles suggesting their reversible characters.
\end{abstract}

Key words: Polymerization, initiator, crosslinker, polyacrylamide hydrogels, ionic salts.

\section{Introduction}

Hydrogels are water swellable, but not soluble, three dimensional cross linked hydrophilic polymer network which can absorb and hold large amount of water that can be much more than thousand times the weight of the polymers. They are inherently soft, hydrophilic, porous and elastic polymer system. In order to keep the spatial structure, the polymer chains are usually physically or chemically crosslinked. The ability of hydrogels to uptake water arises from hydrophilic functional groups such as hydroxilic $(-\mathrm{OH})$, carboxylic (-COOH), amidic (-CONH-), primary amidic $\left(-\mathrm{CONH}_{2}\right)$ and sulphonic $\left(-\mathrm{SO}_{3} \mathrm{H}\right)$ attached to the polymeric backbone. Some hydrophilic monomers commonly used are acrylamide, acrylic acid, methyl methacrylate, isopropyl acrylamide and so on. It is also possible to form hydrogels by copolymerizing hydrophilic and hydrophobic polymers. Some crosslinkers commonly used to

"Corresponding author: Bhadani Reena, Dr., research field: polymer chemistry. crosslink polymeric chains are $\mathrm{N}, \mathrm{N}$-methylene bisacrylamide, 1, 4 butandioledimethacrylate, ethylene glycol dimethacrylate etc [1-8].

Hydrogels responding to external stimuli are often termed as "intelligent or smart hydrogels". The stimuli that influence smart gels are physical and chemical factors. Temperature, light, electric forces, magnetic forces and mechanical forces are types of physical interactions on the gels that precipitate a reaction. Chemical stimuli are usually $\mathrm{pH}$ and chemical environments. The responsive hydrogels have become an important area of research and development in the field of medicine, pharmacy, biotechnology and bioengineering. The renewable, biodegradable, nontoxic various natural polysaccrides including starch, cellulose, sodium alginate, chitosan, guargum etc. are used for the synthesis of environmentally friendly responsive graft copolymer hydrogels with hydrophilic vinyl monomers. Such hydrogels find wide range of application [9-14]. Our some investigations on water swelling behaviour of polyacrylamide hydrogels are discussed in the present paper. 


\section{Experiments}

Acrylamide, N, N'-methylene BAM (Bisacrylamide) and salts were analytical grade and used without further purification. Water was purified by distillation. Pure $\mathrm{NO}_{2}$ was conveniently prepared by heating well dried lead nitrate. Its concentration in $\mathrm{H}_{2} \mathrm{O}$ was determined volumetrically by treating the solution with excess of sodium hydroxide solution and titrated the resulting solution with acidified standard potassium permagnate solution $[15,16]$.

\subsection{Polymerization}

The polymerization of acrylamide was carried out in an aqueous solution of $\mathrm{NO}_{2}$ as an initiator in the presence of BAM at $50{ }^{\circ} \mathrm{C}$ in a glass vessel. When the reaction mixture became very viscous, the polymerization was terminated. The mixture was poured into cold methanol and the polymer was isolated and dried. The polymer so formed was saponified in a known solution of $\mathrm{NaOH}$ for two hours and then precipitated in excess of methanol. The polymers were thoroughly and repeatedly washed with acetone, and dried polymers were stored to determine the degree of swelling.

\subsection{Investigation of Degree of Swelling}

The swelling experiment was carried out by taking a known amount of dry polymer sample and immersed in $200 \mathrm{ml}$ of distilled water to get it hydrated. At a regular interval the swollen gel was taken out and wiped using fine filter paper and weighed. The procedure was repeated until there was no change in the weight of the gel sample. The dried gels are quite hard while swollen gels were very soft and spongy. The degree of swelling was calculated by the following expression:

$$
\text { Degree of Swelling }=\left(\mathrm{m}-\mathrm{m}_{0}\right) / \mathrm{m}_{0}
$$

where, $m_{0}$ is wt. of dry gel and $m$ is wt. of water swollen gel.

\section{Results and Discussion}

\subsection{Swelling Behaviour of Unhydrolysed and Hydrolysed Hydrogel}

The degree of swelling of pure polyacrylamide in water is low compared to that of alkali hydrolysed sample as is seen from Fig. 1

In both cases degree of swelling increases with time and then becomes constant. The unhydrolysed polymer is neutral and has a randomly coiled configuration having a low swelling. On the other hand, hydrolysed polymer has ionic character with an extended chain configuration. Such polymers display a better swelling behaviour.

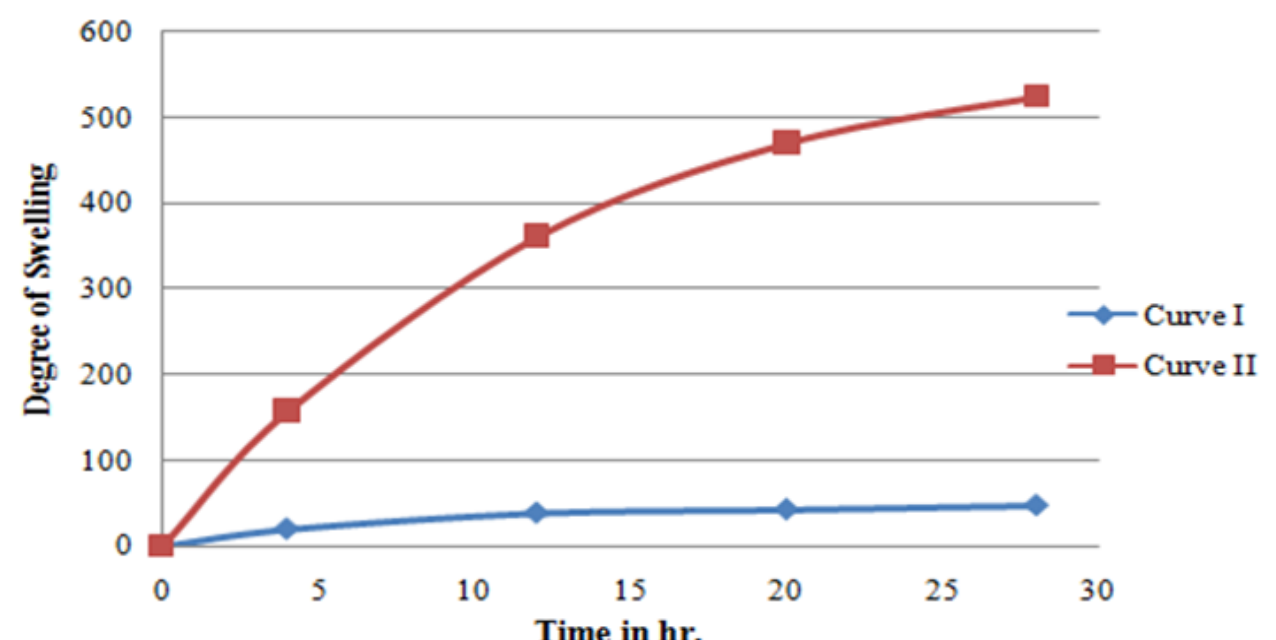

Time in hr.

Fig. 1 Degree of swelling as a function of time for unhydrolysed (Curve I) and hydrolysed (Curve II) ploy (AM) hydrogels in distilled water, wt. of dry unhydrolysed gels and hydrolysed gels is $0.20 \mathrm{gm}$ each, Temp. is $30^{\circ} \mathrm{C}$. 


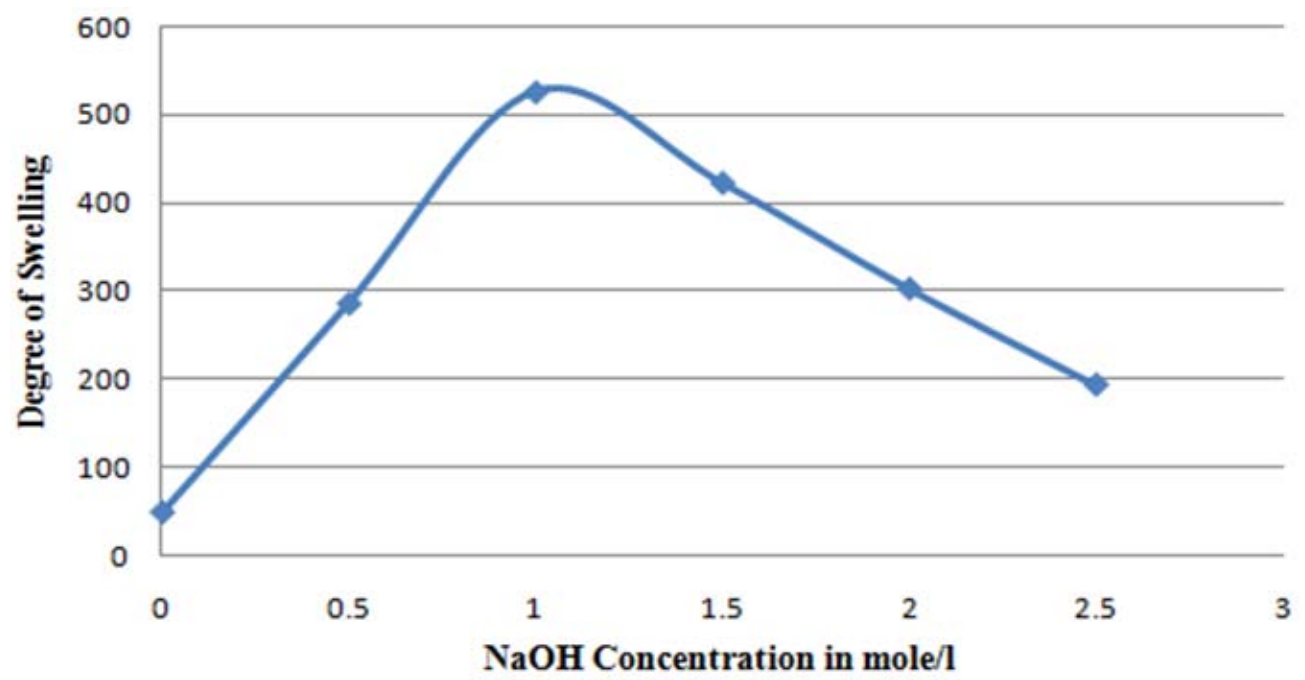

Fig. 2 Degree of swelling vs alkaline hydrolysis as function of different concentration of NaOH. Reaction conditions are i) $[\mathrm{AM}]$ is $6.0 \mathrm{~mol} / \mathrm{l}$, ii) $\left[\mathrm{NO}_{2}\right]$ is $5.5 \times 10^{-2} \mathrm{~mol} / \mathrm{l}$, iii) $[\mathrm{BAM}]$ is $1.5 \times 10^{-2} \mathrm{~mol} / \mathrm{l}$ and iv) polymerization Temp. is $50^{\circ} \mathrm{C}$.

\subsection{Effect of $\mathrm{NaOH}$ Concentration}

The effect of $\mathrm{NaOH}$ concentration, used to hydrolyse polyacrylamide, on the degree of swelling is exhibited in Fig. 2.

The maximum water absorbency is observed at $\mathrm{NaOH}$ concentration of $1 \mathrm{~mol} / \mathrm{l}$ after that it begins to decrease. The hydrolysed polymer contains both amide and carboxyl groups in the chain which are highly polar. The water molecules in the medium are attracted to the polymer molecules by dipole - dipole attraction and hydrogen bonding through nitrogen oxygen and oxygen - oxygen bonding, thereby giving a high degree of swelling. Further increase in $\mathrm{NaOH}$ concentration causes increase in the hydrolysis of the amide groups $\left(-\mathrm{CONH}_{2}\right)$ into the carboxyl group $(-\mathrm{COOH})$ till the equilibrium point is obtained [14]. When the concentration of $\mathrm{NaOH}$ is increased beyond the equilibrium concentration, excess $\mathrm{Na}^{+}$ion in the solution are attracted towards the carboxyl groups in the polymer chain, thus creating effecting local neutral environments along the polymer molecules. This phenomenon is known as charge screening effect and consequently polymer hydrogel configuration is forced to attain some coiled configuration causing deswelling. In addition, a higher crosslinked structure is formed at higher hydroxyl concentration.

\subsection{Effect of Molecular Weights of Polyacrylamide on} Swelling

The polyacrylamide gel synthesized at different concentrations of AM while concentrations other reactants were kept fixed. The gels so obtained were allowed to hydrate and the degree of swelling was determined. The results are exhibited in Fig. 3.

It shows that the decrease in swelling ability with decreasing monomer concentration used during polymer formation. The decreased swelling ability with lower monomer concentration is due to a low molecular weight of the polymer chain. In a free radical polymerization, the molar mass of the resulting polymer increases with monomer concentration. We used $\mathrm{NO}_{2}$ as an initiator to polymerize acrylamide. $\mathrm{NO}_{2}$ molecule contains one unpaired electron shown by its paramagnetism. Therefore, it causes free radical polymerization. So the molecular weight of resulting polymer increased with monomer concentration. It seems swelling ability depends on the molecular weight of polymer chains and longer polymeric chains holds more water.

\subsection{Effect of Concentration of Crosslinker}

The water absorbency of polyacrylamide hydrogels formed at different concentrations of N, N'-methylene 


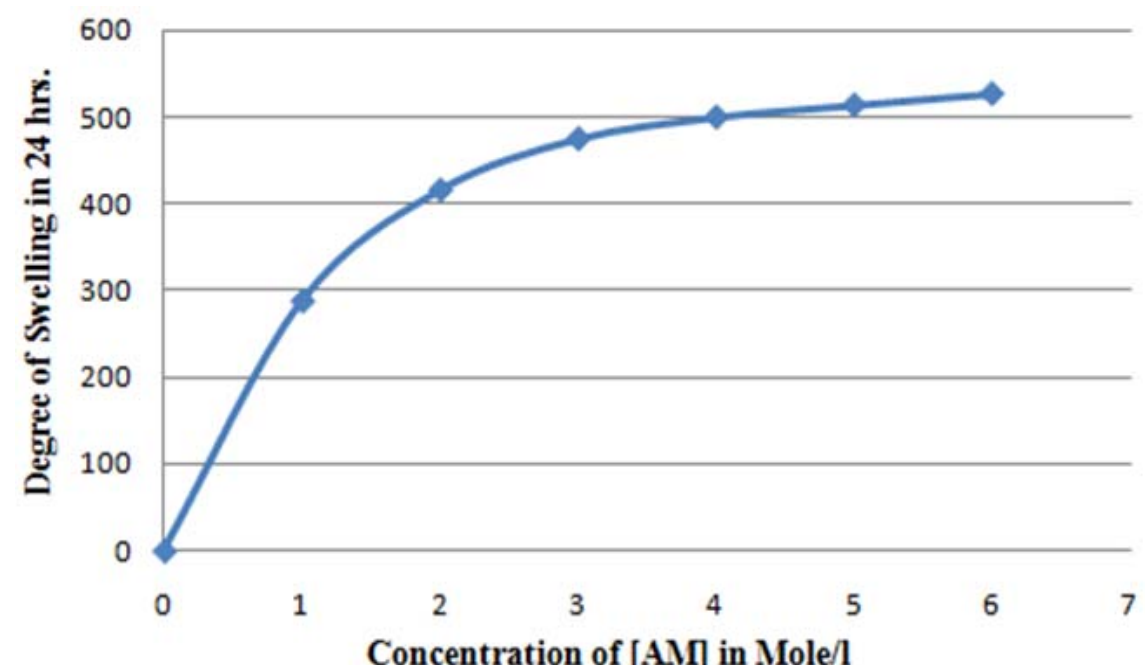

Fig. 3 Degree of swelling in distilled water at $30^{\circ} \mathrm{C}$ as a function of [AM] concentration used in polymerizing mixture to form polyacrylamide hydrogels. Experimental conditions: i) $\left[\mathrm{NO}_{2}\right]$ is $5.5 \times 10^{-2} \mathrm{~mol} / \mathrm{l}$, ii) $[\mathrm{BAM}]$ is $15 \times 10^{-2} \mathrm{~mol} / \mathrm{l}$ and iii) $[\mathrm{NaOH}]$ is $1.0 \mathrm{~mol} / \mathrm{l}$.

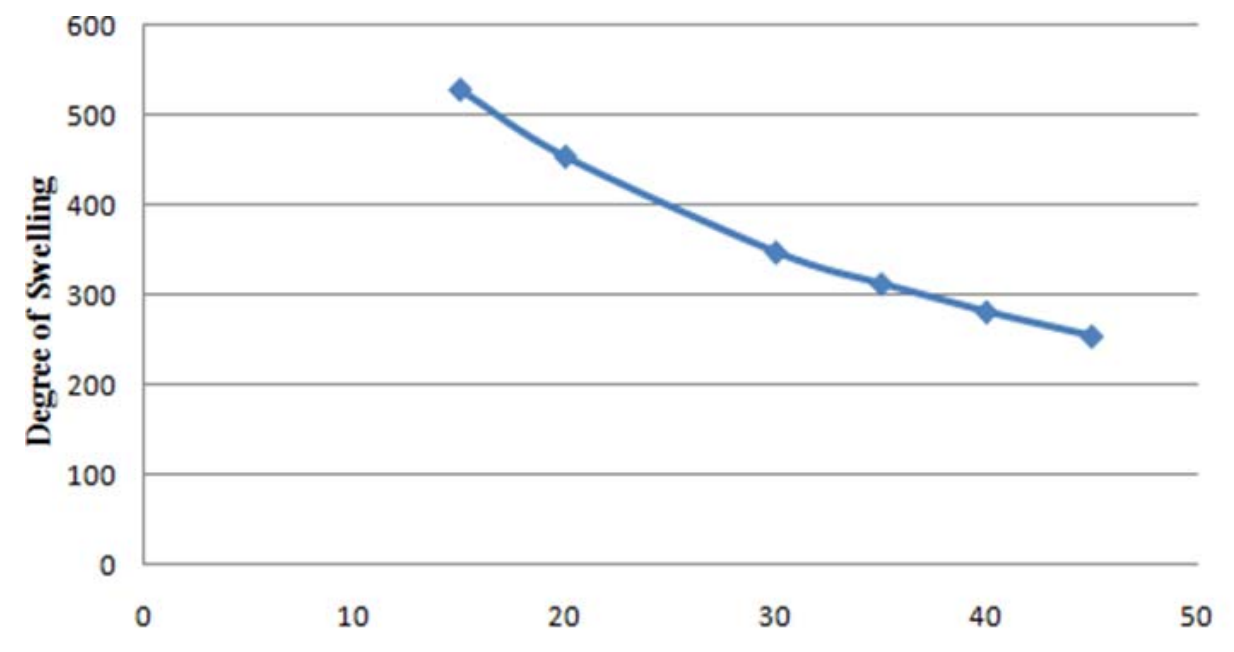

Concentration $[\mathrm{BAM}] \times 10^{2} \mathrm{~mole} / \mathrm{l}$

Fig. 4 Effect of N'-N-methylene bisacrylamide (BAM) concentration on degree of swelling of polyacrylamide hydrogels. Reaction conditions: $[\mathrm{AM}]$ is $6.0 \mathrm{~mol} / \mathrm{l}$, ii) $\left[\mathrm{NO}_{2}\right]$ is $5.5 \times 10^{-2} \mathrm{~mol} / \mathrm{l}$, iii) Reaction medium is $\mathrm{H}_{2} \mathrm{O}$ and iv) Polymerization Temp. is $50{ }^{\circ} \mathrm{C}$.

bisacrylamide (BAM), a crosslinking agent was examined. The pertinent results is presented in Fig. 4.

Fig. 4 shows the degree of swelling decreases with the increase of BAM concentration.

The higher concentration of the crosslinking agent results in the formation of highly crosslinked rigid structure because individual coils are highly tied to each other. Consequently, the gel network is not expanded to uptake a large quantity of water. Therefore increasing degree of crosslinking lowers the swelling ability of gels.

\subsection{Effect of Electrolyte on Swelling}

Result shown in Fig. 5 indicates that the swelling capacity of the hydrogel is significantly lowered by the addition of ionic salts. The deswelling is strongly dependent on kind and concentration of an electrolyte added to the swelling medium. This is possibly due to two effects.

Firstly it is the screening of the fixed charges in the 


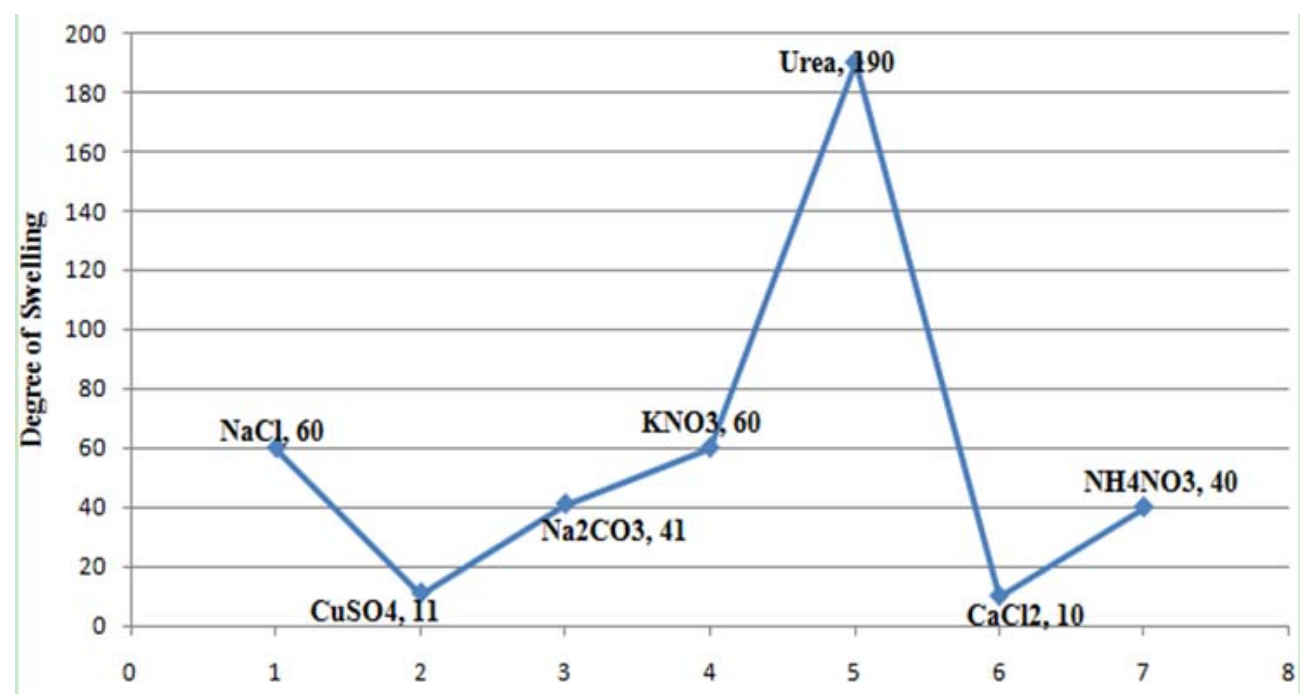

Fig. 5 Effects of salts solution on the degree of swelling of polyacrylamide hydrogels. Concentration of each salts is 0.25 $\mathrm{mol} / \mathrm{l}$ at $30^{\circ} \mathrm{C}$.

polymeric chain and consequently lowering the expansion of the gel network. The negatively charged carboxyl groups attached to polyacrylamide chains set up an anion - anion electrostatic repulsion which tends to expand the network which causes more water uptakes in the hydrogels, when an ionic salts such as $\mathrm{NaCl}$ is added, the presence of $\mathrm{Na}^{+}$ions screen the fixed charges and tremendously reduce the electrostatic repulsion as compared with what it would be in the absence. Therefore the expansion of gel network decreased.

Secondly it is the decrease of the osmotic pressure difference between the gel phase and the external solution phase when ionic strength of the medium increases. The ionic strength of a solution depends on both the mobile ions concentration and their valency as shown by the equation below:

$$
\text { Ionicstrength }=\frac{1}{2} \sum_{i} m_{i} \times Z_{i}^{2}
$$

where, $\mathrm{m}_{\mathrm{i}}$ is molality and $\mathrm{Z}_{\mathrm{i}}$ is valency.

Therefore, (Ionic strength) $)_{\mathrm{NaCl}}=\mathrm{m}, \quad$ (Ionic strength) $)_{\mathrm{Na} 2 \mathrm{SO} 4}=3 \mathrm{~m}$ and (Ionic strength) AlCl3 $_{3}=6 \mathrm{~m}$.

In deed experimental findings show that small quantity of divalent or trivalent ions drastically decrease the degree of swelling. The valence of the accompanying anion does not affect the water absorbency. However the water absorbency capacity of the gel remains unaffected by the urea because of its nondessociating behaviour and having similar functional group $\left(\mathrm{H}_{2} \mathrm{~N}-\mathrm{CO}-\mathrm{NH}_{2}\right)$ as that of acrylamide.

\subsection{Reversibility of Swelling Gels}

The ability of polyacrylamide hydrogel to undergo several cycles of swelling-deswelling is shown in Fig. 6 suggesting its reversible behaviour.

It reveals that in all the successive cycles, it swells slightly more than that of previous cycle. This is due to leaching out some low molecular weight polymer gels. High molecular weight polymer gels show higher degree of swelling. The hydrogels require more time to deswell compared to the time required for swelling.

\section{Proposed Mechanism}

$\mathrm{NO}_{2}$ is an odd-electron molecule and is therefore paramagnetic. Being an odd electron molecule $\mathrm{NO}_{2}$ causes free radical polymerization. The following resonating structure are assigned to this molecule [17]. $\mathrm{NO}_{2}$ interacts with acrylamide to give monomeric free radical which further add monomer molecules to propagate polymerization. 


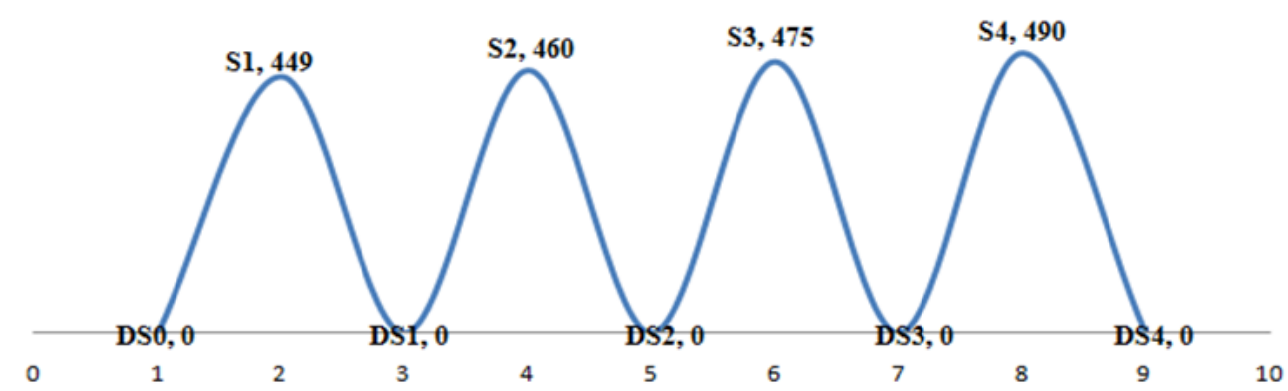

Fig. 6 Swelling (S) and deswelling (DS) cycles of hydrolysed polyacrtlamide hydrogels. Y- co-ordinates represent degree of swelling.

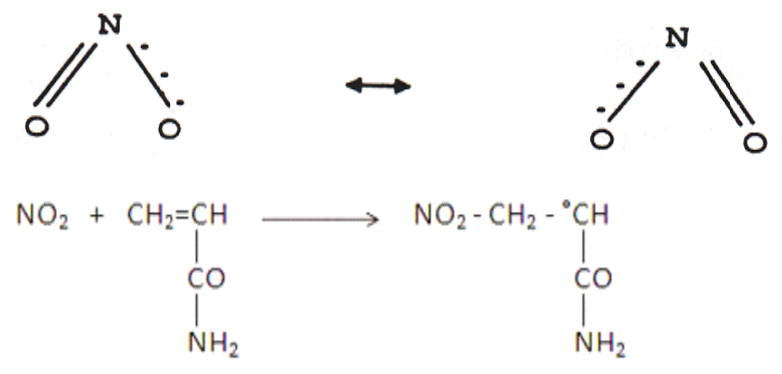

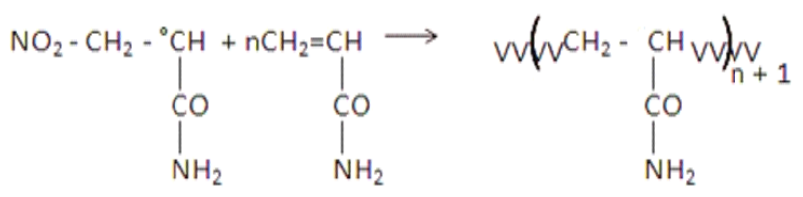

Both monomeric and polymeric units crosslink with bisacrylamide to yield crosslinked polyacrylamide.

\section{Conclusions}

High degree of swelling of polyacrylamide is due to its hydrophilic nature and the capability of hydrogen bonding of acrylamide molecules with water. The molecular size and degree of crosslinking greatly influence the water absorbency of gels.

\section{Acknowledgement}

The financial support of the University Grant Commission, New Delhi to carry out this work is gratefully acknowledged.

\section{Reference}

[1] Flory, P. J. 1964. Swelling of Network Structure, Principle of Polymer Chemistry. Cornell University Press, New York.

[2] Mark, H. F. (Ed.) "Hydrogels in Encyclopaedia of Polymer Science and Technology.” 15: 273-91. Interscience, New York.

[3] Bajpai, S. K. 2001. "Swelling Studies on Hydrogel
Networks -A Review.” J. Sci. Ind. Res. 60 (June): 451-62.

[4] Ganji, F., Vasheghani-Farahani, S. and Vasheghani-Farahani, E. 2010. "Theoretical Description of Hydrogel Swelling: Review.” Iranian Polym. J. 19 (May): 375-98.

[5] Tangri, A. 2014. "Polyacrylamide Based Hydrogels: Synthesis, Characterization and Applications.” Int. J. Pharma. Chem. \&Biolog. Sci. 4 (April): 951-59.

[6] Kundakci, E., Karadag, E. and Uzum, O. B. 2011. "Investigation of Swelling/Sorption Characteristics of Highly Swollen AAm/AMPS Hydrogels and Semi IPNs with PEG as Biopotential Sorbent.” J. Encap. Adsorp. Sci. 1 (March): 7-22.

[7] Sadeghi, M. and Hosseinzadeh, M. 2008. "Synthesis and Swelling Behaviour of Starch - Poly (Sodium Acrylate-Co-Acrylamide) Superabsorbent Hydrogel.” Turkish J. Chem. 32: 375-88.

[8] Das, Nilimanka. 2013. "A Review on Nature and Preparation of Hydrogel Based on Starting Material.” Int. J. Pharmacy Pharmaceut. Sci. 5 (4): 55-8.

[9] Kumar, A. 2011. "Smart Polymeric Biomaterials: Where Chemistry \& Biology can merge.” Indian Institute of Technology, Kanpur. Accessed 2011. http://www.iitk.ac.in/directions/dirnet7/pp Ashok FFF.pdf.

[10] Lim, H. L., Hwang, Y., Kar, M. and Varghese, S. 2014. "Smart Hydrogels as Functional Biomimetic Systems." Biomaterial Sci. 2 (October): 603-18.

[11] Wang, W.-B., Wang, J., Kang, Y.-R. and Wang, A.-Q. 2011. "Synthesis, Swelling and Responsive Properties of a New Composite Hydrogel Based on Hydroxyethyl Cellulose and Medicinal Stone.” Composites: Part B 42 (Jan.): 809-18.

[12] Sadeghi, M. and Hosseinzadeh, M. 2013. "Synthesis and Properties of Collagen - g - Poly (Sodium Acrylate - co 2 - Hydroxyethylacrylate) Superabsorbent Hydrogels.” Braz. J. Chem. Eng. 30 (June): 1-11.

[13] Dogu, Y. and Okay, O. 2005. "Swelling - Deswelling Kinetics of Poly (N - isopropylacrylamide) Hydrogels Formed in PEG Solutions." J. App. Polym. Sci. 99 (Jan.): 37-44.

[14] Bhadani, R. and Mitra, U. K. 2014. "Synthesis and 
Characterization of Polyacrylamide Hydrogels.” Asian J. Res. Chem. 7 (March): 345-8.

[15] Vogel, A. I. 1969. “Quantitative Inorganic Analysis.” 3rd Edition. GBLS and Longman. London. 297.

[16] Bhadani, S. N. and Prasad, Y. K. 1980. "Kinetics of
Nitrogen Dioxide Initiated Polymerization of Acrylamide.” Makromol. Chem. 181: 1089-95.

[17] Bhadani, S. N. and Sen Gupta, S. K. 1996. "Gaseous Initiator Polymerization.” Polymer Material Encyclopaedia. CRC Press. U. S. A. 22-7. 